\title{
KINETIC THEORY OF PLASMA WAVES
}

\author{
D. Van Eester and E. Lerche \\ Laboratorium voor Plasmafysica - Laboratoire de Physique des Plasmas \\ EUROfusion Consortium member \\ Koninklijke Militaire School - Ecole Royale Militaire \\ Trilateral Euregio Cluster, Renaissancelaan 30 - B1000 Brussels - Belgium \\ Tel.: (32 2) 4414 134,Fax.: (32 2)735 2421, e-mail: d.van.eester@fz-juelich.de8 ealerche@msn.com
}

\begin{abstract}
In the present paper a very brief introduction is provided to the theory of kinetic waves relevant to the description of wave heating in fusion machines and focussing mostly on radio frequency or ion cyclotron resonance frequency waves in tokamaks. The text starts by sketching the basic philosophy underlying the standardly adopted methods, describing the interaction of a single particle with a given wave and the assumptions typically made to arrive at a trustworthy description of the energy exchange, and ends by discussing some of the subtleties of the modeling of wave-particle interaction in inhomogeneous magnetized plasmas. None of the topics will be treated in full detail. Hence, by no means, this text is meant to be all-inclusive. Rather, it aims at providing a framework that should allow understanding what are the difficulties involved, leaving out the detailed derivation of the expressions as well as subtleties such as relativistic corrections. The interested reader is referred to the provided references - and the references given therein - for more in depth information.
\end{abstract}

\section{INTRODUCTION}

The interaction between charged particles and electromagnetic waves can be looked at from 2 vantage points: From the point of view of the waves 'plasma heating' is a process by which they lose energy. The relevant equation to describe this is the wave equation, derived from Maxwell's equations. From the point of view of the particles the same process is viewed as a gain of energy. The relevant equation to describe this second interpretation of the same physical phenomenon is the Fokker-Planck equation, derived starting from the kinetic equation of state. A proper description of the phenomena requires that these 2 aspects are described on the same footing, which is not at all straightforward and which only starts to be done now that powerful computers are increasingly available.

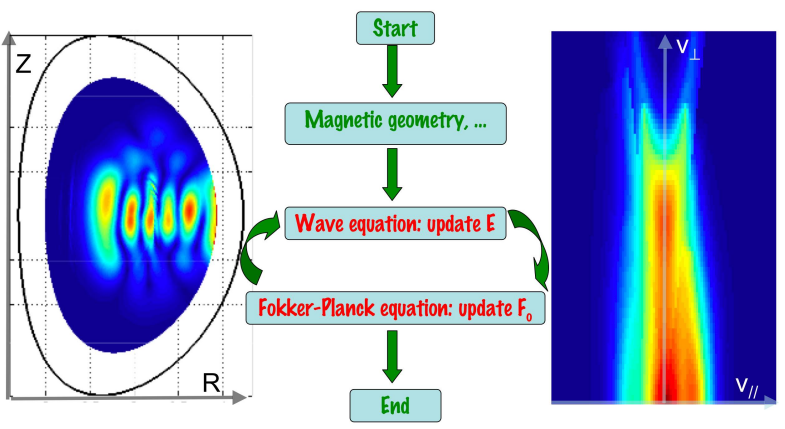

Figure 1: Wave-particle interaction: wave point of view (left: fast dynamics) vs. particle point of view (right; slow - net - dynamics) and scheme for modeling both aspects self-consistently. The wave field (left) is plotted in a toroidal cut of a tokamak, the RF heated ion distribution (right) is plotted in terms of the velocity components at the low field side crossing of the equatorial plane for a prescribed radial position.

The kinetic description of waves in plasmas typically starts from the equation

$$
\frac{d f}{d t}=C+S-L
$$

in which $f$ is the distribution function of the charged particles being studied, and the right hand side describes how the distribution equation evolves under the influence of collisions the particles undergo, and of particle injection ( $\mathrm{S}=$ source) and particle loss $(\mathrm{L})$. In the high frequency domain and for a sufficiently strong magnetic field, the left hand side is dominated by processes on a vastly different time scale than that of the net effect of collisional interaction and particle loss or gain. Hence on the fastest time scale of the problem the right hand side is negligibly small and can be neglected to a first approximation. The above equation then simply states that the number of particles is conserved in phase space: Particles can move about and gain or lose energy - which causes a stretching or squeezing of the 
volume in which a given number of particles resided at a given initial time - but the number of particles in the stretched phase-space volume is always conserved.

\section{BASIC PHILOSOPHY [1-11]}

\section{II.A. General Formulation}

In general, the orbits of particles immersed in electromagnetic fields are not integrable i.e. their motion cannot be described in terms of constants of the motion but is stochastic and thus ergodically covering parts of phase space. On top of that, charged particles in motion constitute a current themselves and thus influence the electromagnetic fields in the fusion machine. Hence, the RF plasma current needs to be carefully accounted for when solving Maxwell's equations. Describing the impact of the charged particles on the fields and the back reaction of the fields on the particles involves the challenging task of solving a set of coupled nonlinear equations in 6 independent variables in phase space. As the temporal and spatial scales cover a range of many orders of magnitude (ion cyclotron motion involves frequencies in the radio frequency - megaHertz - domain while net collisional interaction occurs on a time scale of hundreds of milliseconds in a tokamak such as JET, or seconds in ITER; the macroscopic dimensions of such fusion machines is several meters, while the ion Larmor radius $\rho$ is of the order of a few millimeters) making simplifications is a necessity. The drawback of the wide range of scales is thereby turned into an advantage, allowing to set apart phenomena and tackling processes happening on drastically different scales separately.

It is instructive to have an idea of the relative magnitudes of various relevant quantities to understand why the 'quasi-linear' approach and other commonly made approximations make sense. For typical JET parameters in a D majority plasma (temperature of $5 \mathrm{keV}$, density of $5 \times 10^{19} \mathrm{~m}^{-3}$, magnetic field $3 T$, major radius of $3 \mathrm{~m}$ and minor radius of $1 \mathrm{~m}$ ), the ion cyclotron frequency of the $\mathrm{D}$ ions is $23 \mathrm{MHz}$ and the electron cyclotron frequency is $80 \mathrm{GHz}$, the ion thermal velocity is $5 \times 10^{5} \mathrm{~m} / \mathrm{s}$ so the typical ion Larmor radius is $3 \mathrm{~mm}$, the electron thermal velocity $3 \times 10^{7} \mathrm{~m} / \mathrm{s}$ so the electron gyroradius is $0.05 \mathrm{~mm}$, the ion collision frequency is $100 \mathrm{~Hz}$ and the electron collision frequency is $10 \mathrm{kHz}$. For typical RF waves of several $M W$ with electric field values of $50 \mathrm{kV} / \mathrm{m}$ close to the antennas, the RF magnetic field is $5 \times 10^{-3} \mathrm{~T}$ and the RF magnetic contribution to the Lorentz acceleration $\left|\vec{v} \times \vec{B}_{R F}\right|=2.5 \mathrm{kV} / \mathrm{m}$.

Hence the ions travel around the torus in about $4 \times 10^{-5} s$, the cyclotron ('gyro') period $\tau_{g}$ being much shorter than the transit ('bounce' \& 'drift') time $\tau_{b, d}$, which itself is much smaller than the collision time $\tau_{c}$, making it senseful to describe the cyclotron motion as much faster than the bounce/transit motion, itself typically much faster than the collision time and rendering a collisionless description senseful. The scaling $\tau_{g}<<\tau_{b, d}<<\tau_{c}$ is crucial for the customary models. In particular, the 'slower' phenomena are assumed to be constant on the faster time scale while the faster - oscillatory - phenomena are treated as being beyond their transient state, all quantities merely varying as a function of time as $\exp [i \omega t]$, where $\omega$ is the frequency at which the external wave launchers are operated. The particle motion is essentially imposed by the confining magnetic field, the RF field being a small - be it fast perturbation and the RF electric field effect dominating that of the magnetic field. Finally, the Larmor radius is commonly much smaller than the equilibrium quantity gradients, this giving rise to the so-called drift approximation and locally making a quasi-homogeneous description senseful. In particular $\rho / L_{B_{o}}<<1$ where $\rho$ is the Larmor radius and $L_{B_{o}}$ is a typical scalelength of the variation of the confining magnetic field.

To understand the basic physics of the impact of the RF electric field on a test particle, we locally solve the equation of motion and then use the result to evaluate the net energy a charged particle can gain or lose in a rapidly varying electric field along the trajectory it is forced to follow by the fusion machine's static confining magnetic field. We start from a homogeneous plasma, straight magnetic field line analysis and gradually include other effects.

Because the magnetic field is imposing a clear asymmetry in the dynamics along as opposed to perpendicular to the magnetic field lines, the discussion of the wave-particle interaction is most easily described with reference to the direction along $\vec{e}_{/ /}=\vec{B}_{o} / B_{o}$ and 2 independent directions perpendicular to the static magnetic field $\vec{B}_{o}$. Neglecting the equilibrium electric field related to the ohmic circuit, the solution of the equation of motion of a charged particle immersed in a homogeneous, static magnetic field can be written as $v_{\perp, 1}=v_{\perp} \cos \phi, v_{\perp, 2}=v_{\perp} \sin \phi$ where $\phi=\phi_{o}-\Omega\left(t-t_{o}\right)$ while $v_{/ /}=c t$, with $\Omega=q B_{o} / m$ ( $B_{o}$ the confining field, $q$ the charge and $m$ the mass of the species) the cyclotron frequency, which can further be integrated to get the particle position: $x_{\perp, 1}=x_{\perp, 1, G C}-\rho \sin \phi$, $x_{\perp, 2}=x_{\perp, 2, G C}+\rho \cos \phi$ in which the Larmor radius is given by $\rho=v_{\perp} / \Omega$ and 'GC' refers to the guiding centre position. Assuming the electric field is a plane wave characterized by a wave vector $\vec{k}$, defining $\psi$ as the angle between $\vec{k}$ and $\vec{e}_{\perp, 1}\left(k_{\perp, 1}=k_{\perp} \cos \psi, k_{\perp, 2}=k_{\perp} \sin \psi\right)$, the work the electric field does on a particle can be written

$$
q \vec{E} \cdot \vec{v}=q \sum_{N=-\infty}^{N=+\infty} L_{N} \exp [-N \phi]
$$


in which

$$
\begin{aligned}
& L_{N}=\left[\frac{v_{\perp}}{2}\left(E_{-} J_{N+1} e^{i \psi}+E_{+} J_{N-1} e^{-i \psi}\right)\right. \\
& \left.+E_{/ /} v_{/ /} J_{N}\right] e^{i N \psi}
\end{aligned}
$$

is the Kennel-Engelmann operator [17] and where the electric field is evaluated at the guiding center $\vec{x}_{G C}=$ $\left[x_{\perp, 1}+\rho \sin \phi\right] \vec{e}_{\perp, 1}+\left[x_{\perp, 2}-\rho \cos \phi\right] \vec{e}_{\perp, 2}+\left[v_{/ /}\left(t-t_{o}\right)\right] \vec{e}_{/ /}$ rather than at the particle position and in which the argument of the Bessel functions is $k_{\perp} \rho$. In doing so the most rapidly varying contribution (the cyclotron oscillation) is isolated from all slower contributions. Figure 2 illustrates that using the guiding center position rather than the particle position as the reference position makes the bookkeeping much simpler when studying heating: In the particle frame, integration over a reference volume entails integrating over all orbits with various speeds and guiding centers that are intersecting the reference volume. When particles are in coherent motion with a wave and are periodically exchanging energy with it, this exchange is not considered to be 'heating' although the energy streaming into the reference volume in $\vec{x}$ will increase at some times and decrease at others. In the guiding center $\vec{x}_{G C}$ frame the picture is much clearer, simpler and more symmetrical, as there is no leaking of particles into or out of reference volumes. On top of that, the fastest evolution has been separated out, a non-negligible advantage when searching for equations that will need to be solved numerically as it implies a significant speed-up of the computations. Finally, as will be seen later, expressing the fields in terms of guiding center coordinates allows interfacing to the Fokker-Planck equation describing the net impact of the fields on the particles (rather than the impact of the particle motion on the fields) in a natural way, allowing to make wave and particle equations more easily compatible. From the equation of motion one readily finds that the change of the particle energy is $\dot{\epsilon}=\frac{d \epsilon}{d t}=q \vec{E} \cdot \vec{v}$ which, using the above found expression, can be written more explicitly as

$\dot{\epsilon}=q \sum_{-\infty}^{+\infty} L_{N}\left(\vec{E}_{G C}\left(t_{o}\right)\right) \exp \left[i\left(N \Omega+k_{/ /} v_{/ /}-\omega\right)\left(t-t_{o}\right)\right]$.

For most frequencies $\omega$ the right hand side is periodic and hence the energy transfer between the electric field and the particles is merely oscillating around an average value but no net acceleration is taking place. At the Doppler shifted cyclotron resonances $\omega=N \Omega+k_{/ /} v_{/ /}$ the exponential time dependent factor associated to a specific cyclotron harmonic $N$ on the right hand side is constant and hence - in spite of all other terms still oscillating as a function of time - there is a net energy transfer.
Net heating takes place when $N \Omega+k_{/ /} v_{/ /}=\omega$, in which the Doppler shift term $k_{/ /} v_{/ /}$is usually a correction to $N \Omega$, except when $N=0$ in which case it is crucial. In the radio frequency domain (tens of $\mathrm{MHz}$ ) and for typical magnetic field strengths of current-day magnetic fusion machines (a few Tesla), the resonance condition for the ions can easily be satisfied for $N \neq 0$ i.e. they undergo cyclotron heating, while that of the electrons requires $N=0$ i.e. they feel the Cerenkov effect. As a consequence, ions and electrons react very differently to waves driven at frequencies in the ion cyclotron frequency range: For not too energetic particles, the argument $k_{\perp} \rho$ of the Bessel function is small so that $J_{0} \approx 1$ and $J_{M}<<1$ when $M \neq 0$. Hence, the ions are mainly accelerated in the perpendicular direction by the perpendicular components of the electric field while the contribution of the parallel electric field has a minor impact on them; on the other hand, the parallel electric field gives the electrons a net pull in the parallel direction (Landau damping). Cerenkov interaction equally involves the perpendicular electric field components, an effect known as transit time magnetic pumping (TTMP). Whereas Landau damping causes parallel acceleration and is present even when the electric field is spatially uniform, TTMP affects the perpendicular energy and requires inhomogeneity of the field. An elegant discussion of the wave-particle interaction can be found in [12].

Since collisons are infrequent but non-absent, it is customary to interpret the frequency $\omega$ in the resonant denominator as a complex quantity with a very small, positive imaginary part $i \nu, \nu$ loosely being interpreted as the collision frequency that would appear in the particle equation of motion if collisions would be accounted for in a simple way. This gives a recipe for how to encircle the poles at the resonances to ensure causality. The contribution of the energy from events in the far past $\left(t_{o} \rightarrow-\infty\right)$ is then absent and only the end contribution of the time integral at time $t$ survives. The need for the elimination of the far past history is of particular interest to ensure there is net heating. It will be discussed separately later.

II.B. The Quasilinear Approach: The RF Perturbed Distribution and the Quasilinear Diffusion Operator

The time evolution equation (1) is rewritten making use of the fact the confining magnetic field is much larger than the fastly varying purely oscillatory electromagnetic perturbation, driven at the antenna frequency $\omega$ i.e. proportional to $\propto \exp [i \omega t]$ : Both the distribution itself and the Lorentz force are separated into a large term only involving slowly varying quantities (referred to with a subscript 'o'), and a small but rapidly varying 


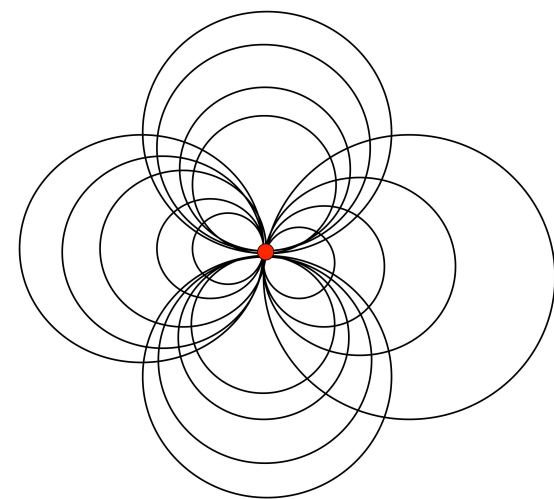

Particle position as reference position: all orbits crossing through a given point $\odot$

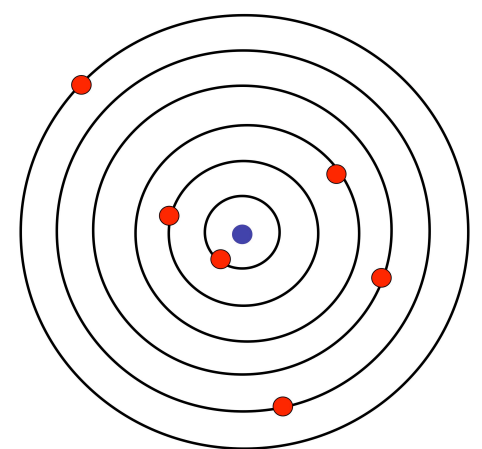

Guiding center position as reference position: all orbits sharing the same guiding center

Figure 2: Cyclotron motion as seen in the particle (top) and guiding centre (bottom) reference frame.

contribution (related to the driven RF fields):

$$
\begin{gathered}
\frac{d f}{d t}=\left.\frac{d f}{d t}\right|_{o}+\left.\frac{d f}{d t}\right|_{R F}= \\
\left.\frac{d F_{o}}{d t}\right|_{o}+\left.\frac{d F_{o}}{d t}\right|_{R F}+\left.\frac{d f_{R F}}{d t}\right|_{o}+\left.\frac{d f_{R F}}{d t}\right|_{R F}=C+S-L
\end{gathered}
$$

with $\left.\frac{d}{d t}\right|_{o}=\frac{\partial}{\partial t}+\vec{v} \cdot \nabla_{\vec{x}}+\frac{q}{m}\left[\vec{E}_{o}+\vec{v} \times \vec{B}_{o}\right] \cdot \nabla_{\vec{v}}$ and $\left.\frac{d}{d t}\right|_{R F}=\vec{a}_{R F} \cdot \nabla_{\vec{v}}=\frac{q}{m}\left[\vec{E}_{R F}+\vec{v} \times \vec{B}_{R F}\right] . \nabla_{\vec{v}}, F_{o}$ the slowly varying and $f_{R F}$ the rapidly varying distribution function. The first, zero order term in the above only varies on the slowest time scale, the next 2 terms are first order corrections which oscillate at frequency $\omega$, while the most rapidly varying terms in the last, second order term contains factors that oscillate at frequency $2 \omega$. Since $F_{o}$ only depends on the constants of the motion, $\left.\frac{d F_{o}}{d t}\right|_{o}$ can be simplified to $\frac{\partial F_{o}}{\partial t}$. The 2 linear terms yield an expression for the RF perturbed distribution i.e. for the evolution on the fast time scale, known as the Vlasov equation:

$$
\vec{a}_{R F} \cdot \nabla_{\vec{v}} F_{o}+\left.\frac{d f_{R F}}{d t}\right|_{o}=0
$$

i.e.

$$
f_{R F}=-\int_{o r b i t}^{t} d t^{\prime} \vec{a}_{R F} \cdot \nabla_{\vec{v}} F_{o}
$$

which can be inserted in the fourth term of the evolution equation. Averaging $\langle\ldots\rangle$ the 4 terms over a full oscillation period for all oscillatory aspects of the motion and the driven response, yields an equation for the slow time variation, known as the Fokker-Planck equation. The first term stays untouched, the second and third term as well as the oscillatory parts of the fourth term vanish while a constant, second order contribution survives. This yields

$$
\frac{\partial F_{o}}{\partial t}=<C>+<S>-<L>+<Q>
$$

in which $<Q>=<\nabla_{\vec{v}} \cdot \vec{a}_{R F}^{*} \int_{o r b i t}^{t} d t^{\prime} \vec{a}_{R F} \cdot \nabla_{\vec{v}} F_{o}>$ is the quasi-linear diffusion operator, acting on the slowly varying distribution function.

\section{II.C. The Wave Equation \& The Conductivity Tensor}

Combining Maxwell's equations for the evolution of the electric field and the magnetic field, and assuming the waves are driven at a frequency $\omega$, the wave equation can be written in terms of the electric field $\vec{E}$ only,

$$
\nabla \times \nabla \times \vec{E}-k_{o}^{2} \vec{E}=i \omega \mu_{o}\left[\vec{J}_{\text {antenna }}+\vec{J}_{\text {plasma }}\right],
$$

in which $k_{o}=\omega / c$ with $c$ the speed of light. The fields are excited by the current density $\vec{J}_{\text {antenna }}$ flowing on the antennas typically located close to the edge of the plasma. The plasma current $\vec{J}_{\text {plasma }}$ is composed of the contributions from the various plasma constituants $s$, $\vec{J}_{\text {plasma }}=\sum_{s} q_{\alpha} \int d \vec{v} \vec{v} f_{R F, s}$, and is fully defined when the perturbed distributions of all species are known. Strictly, the plasma current contains an ohmic contribution $\left(\vec{J}_{\text {plasma }}=\vec{J}_{\text {ohmic }}+\vec{J}_{R F}\right)$ aside from the fastvarying $R F$ contribution. It has been neglected in the present text.

An elegant way to solve the wave equation is relying on variational techniques, by multiplying the equation with a test function vector and integrating over the volume of interest. Performing partial integration to remove the highest order derivatives from $\vec{E}$ not only allows to chose lower order base functions for a given desired numerical accuracy when solving the equation, it also allows to obtain a more symmetrical formulation in which the test function vector $\vec{F}$ and the electric field $\vec{E}$ play a similar role. The resulting equation is

$$
\begin{gathered}
\int d \vec{x}\left[k_{o}^{2} \vec{F}^{*} \cdot \vec{E}-(\nabla \times \vec{F})^{*} \cdot(\nabla \times \vec{E})\right]+W= \\
-\left[\int_{\text {surface }} d \vec{S} \cdot \vec{F}^{*} \times \nabla \times \vec{E}+i \omega \mu_{o} \int d \vec{x} \vec{F}^{*} . \vec{J}_{\text {antenna }}\right]
\end{gathered}
$$


with $W /\left[i \omega \mu_{o}\right]=\int d \vec{x} \vec{F}^{*} \cdot \vec{J}_{R F}=q \int d \vec{x} d \vec{v} \vec{F}^{*} \cdot \vec{v} f_{R F}$. The surface term needs to vanish at the metallic wall to ensure no electromagnetic flux leaks away. A supplementary advantage of this formulation is that it readily yields the associated energy conservation theorem when substituting the test function vector by the electric field (see further for the expression for the absorbed power density shared by the wave and particle descriptions). The perturbed current density $\vec{J}_{\text {plasma }}$ and the electric field $\vec{E}$ are related by the conductivity tensor $\overrightarrow{\vec{\sigma}}$ : In Fourier space $\vec{J}_{\text {plasma }, \vec{k}^{\prime}}=\overrightarrow{\vec{\sigma}}_{\vec{k}^{\prime}, \vec{k}} \cdot \vec{E}_{\vec{k}}$ which is closely related to the dielectric tensor $\overrightarrow{\vec{K}}=\overrightarrow{\overrightarrow{1}}+i \omega \mu_{o} \overrightarrow{\vec{\sigma}}$.

For a plasma in thermal equilibrium, the term $q \vec{F}^{*} \cdot \vec{v} f_{R F}$ can be written more explicitly as $-q \vec{F}^{*} \cdot \vec{v} \int^{t} d t^{\prime} q \vec{E} \cdot \vec{v} \frac{F_{o}}{k T}$ in which the last factor can be shifted in front of the particle history integral since the slowly varying distribution only depends on the constants of the motion. One gets

$W=\omega \mu_{o} q^{2} 2 \pi \int d \vec{x} d v_{\perp} d v_{/ /} v_{\perp} \frac{F_{o}}{k T} \sum_{N} \frac{L_{N}(\vec{F})^{*} L_{N}(\vec{E})}{N \Omega+k_{/ /} v_{/ /}-\omega}$

Isolating the various contributions from the test function vector and the electric field in this expression yields an expression for the conductivity tensor.

The velocity space integrals in Eq. (6) can be performed to yield a compact expression for the dielectric response in a Maxwellian plasma. The integral over the parallel velocity yields the Fried-Conte plasma dispersion function $Z(\zeta)$, which - aside from the hot plasma corrections to the wave propagation describes the process of collisionless damping. The argument of the Fried-Conte function is $\zeta=\frac{\omega-N \Omega}{k_{/ /} v_{t h}}$. Figure 3 depicts this function for $\operatorname{Im}(\zeta) \rightarrow 0^{+}$. The real part asymptotically approaches the cold plasma limit $R e[Z] \approx-1 / \zeta$, but bends the resonant crossing from $+\infty$ to $-\infty$ at $\zeta=0$ into a smooth transition behaving like $R e[Z] \approx-2 \zeta$. The imaginary part is a Gaussian. Physically its width is determined by the scalelength over which the cyclotron frequency $\Omega$ varies, and the factors contributing to the Doppler shift, namely the parallel wave number $k_{/ /}$and the thermal velocity $v_{t h}$. Away from the cold plasma resonance damping fades away quickly while the reactive part stays significant much further from $\zeta=0$.

As long as $k_{\perp} \rho<<1$ is satisfied, the Bessel functions can easily be approached by their truncated Taylor series expansion and the perpendicular integrals can easily be integrated. Retaining all finite Larmor radius effects yields modified Bessel functions (see e.g. [3]). Although the perpendicular (cyclotron gyration) dynamics seems more daunting than the parallel dynamics, it is the latter that is most challenging: In strong magnetic fields, the cyclotron motion moves the particle only a small distance - the Larmor radius $\rho$ - away from the guiding center, hence equilibrium quantities typically vary little between the particle and the guiding center positions. But the guiding centers themselves often sample large regions of the machine since their motion is only restricted by the magnetic field topology. Taylor series expansions are routinely used for the perpendicular dynamics but have to be used with care for the parallel dynamics.

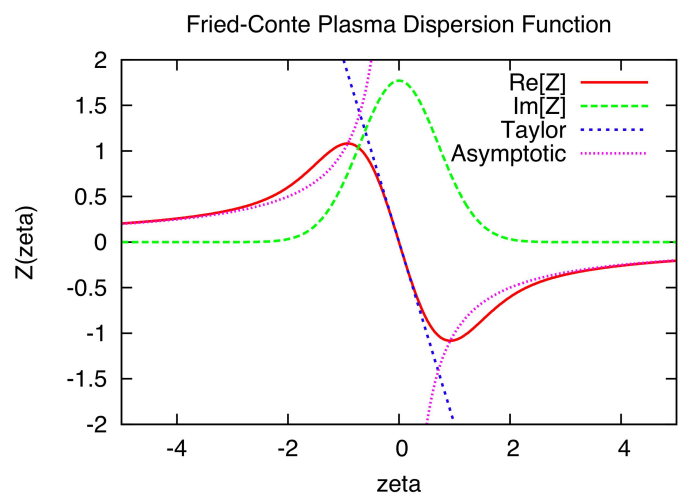

Figure 3: The Fried-Conte plasma dispersion function and its leading order Taylor and asymptotic series representation.

Expressions have also been derived to account for arbitrary $F_{o}$ (see e.g. [7]). The Fried-Conte function is now replaced by other (in general numerically evaluated) functions. For a sufficiently refined velocity grid, the distribution function can locally be approximated with bi-linear functions and the partial integral can be evaluated analytically, yielding a logarithmic contribution. Upon crossing the resonance, the logarithm picks up a 'switch-on kick' imaginary contribution: It is the delta function contribution at the pole of the original integrand that represents the discontinuous Heaviside step energy 'kick' when picking up the energy due to crossing the resonance. The kick shows up in the uniform plasma description as a resonance crossing in velocity space. In non-uniform plasmas the kick can just as well be described by integrating along the orbit.

\section{II.D. The Cold Plasma Limit}

To get a feeling of how drastically a plasma changes the wave characteristics of the electromagnetic waves that exist in vacuum, it is already sufficient to simply consider the cold plasma limit. This may seem a drastic oversimplification but since $k_{\perp} \rho$ is small when the temperature is low, the cold plasma limit yields a reasonable description of the fate of the waves launched from RF antennas, to the obvious exception of the collisionless damping processes themselves which are an 
inherently kinetic - as opposed to fluid - effect.

Although it is sufficient to take the asymptotic limit $Z(\zeta) \rightarrow-1 / \zeta$ and $J_{0}\left(k_{\perp} \rho\right) \rightarrow 1$, while $J_{M} \rightarrow 0$ for Bessel function with order $M>1$ to retrieve the cold plasma limit, it is much easier to directly rederive the conductivity tensor starting from the solutions of the equation of motion. Using the Stix notation [3], the cold plasma dielectric tensor can be written

$$
\overrightarrow{\vec{K}} \cdot \vec{E}=\left(\begin{array}{ccc}
S & -i D & 0 \\
i D & S & 0 \\
0 & 0 & P
\end{array}\right) \cdot\left(\begin{array}{c}
E_{\perp, 1} \\
E_{\perp, 2} \\
E_{/ /}
\end{array}\right)
$$

in which $S=(R+L) / 2, D=(R-L) / 2$, with

$$
\begin{gathered}
R=1-\sum_{s} \omega_{p, s}^{2} / \omega\left(\omega+\Omega_{s}\right), \\
L=1-\sum_{s} \omega_{p, s}^{2} / \omega\left(\omega-\Omega_{s}\right) \\
P=1-\sum_{s} \omega_{p, s}^{2} / \omega^{2}
\end{gathered}
$$

where the sum is on the various types of species $s$ the plasma is constituted of and $\omega_{p}$ is the plasma frequency.

\section{II.E. Dispersion Equation Roots}

Waves in a cold plasma are electromagnetic in character i.e. their energy is carried purely by the Poynting flux. When the plasma density goes to zero, their dispersion roots join the vacuum roots $k_{\perp}^{2}=k_{o}^{2}-k_{/ /}^{2}$. With respect to $\vec{B}_{o}$, one of the 2 cold plasma roots is essentially transverse electric, and the other essentially transverse magnetic in character. Referring to the group (energy propagation) velocity, the former is known as a 'fast' wave while the other is a 'slow' wave. The former allows to carry wave power across magnetic surfaces and is the preferred candidate to heat the plasma core in the ion cyclotron domain, while the latter tends to propagate along magnetic surfaces. Finite temperature effects add kinetic corrections to these modes, and introduce supplementary wave branches. For not too energetic particles, the dielectric tensor is usually truncated at second order effects in the Larmor radius. This results in a supplementary mode appearing in the dispersion equation: the (first) Bernstein wave. This wave is essentially electrostatic in nature i.e. its energy is carried by particles in coherent motion with the wave, while its Poynting flux is negligible. Figure 4 shows a dispersion plot of the fast wave exciting the Berstein wave at the place where the decoupled cold plasma fast wave has a resonance $\left(S=k_{/ /}^{2}\right)$. This being very close to the ion-ion hybrid layer $(S=0)$ since $k_{/ /}^{2}<<|S|$ in sufficiently dense plasmas, the mode conversion layer is often labeled as the ion-ion hybrid layer.

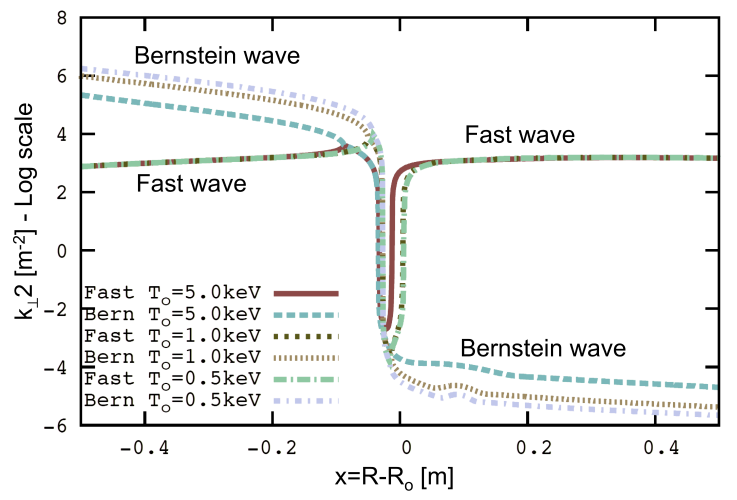

Figure 4: Fast and (first) ion Bernstein wave dispersion equation roots for 3 different central temperatures using a dielectric description retaining all finite Larmor radius corrections. Note that the fast wave root hardly changes while the Bernstein wave root - a root absent in a cold plasma description - depends sensitively on the temperature.

Strictly speaking, the Berstein wave cannot be described by a dispersion resulting from a truncated Taylor series expansion in $k_{\perp} \rho$ since $k_{\perp, B e r n} \rho$ is of order 1 , although such a model does correctly locate the places where the fast wave excites it for up to second cyclotron harmonic terms. At higher frequencies and/or for more energetic particles, the customary truncation of the dielectric tensor is not even rigorous for the fast wave anymore. Hence, higher order finite Larmor radius terms have to be retained. A hot plasma supports an infinity of hot plasma modes, adding supplementary Bernstein modes. Whether they actually play a role depends on whether or not they are excited. Increasing the frequency while keeping the magnetic field fixed brings higher harmonics into the plasma. Higher Bernstein wave modes can be excited but the fast and Bernstein waves are gradually more decoupled at higher frequencies.

\section{II.F. The Fokker-Planck Equation [17-23]}

Electromagnetic waves cannot directly be observed experimentally so their behavior is indirectly studied through e.g. the response of temperature and density to sudden changes in the externally launched power level (see e.g. [22]). On the other hand, multiple diagnostics exist to monitor aspects of fast particle populations present in the plasma and to cross-check against theoretical predictions.

As briefly discussed before, when all fast scale dynamics are removed from the description by averaging over all oscillatory aspects of the motion and driven response, the Fokker-Planck equation (4) results. Whereas the wave equation is commonly tackled by integrating 


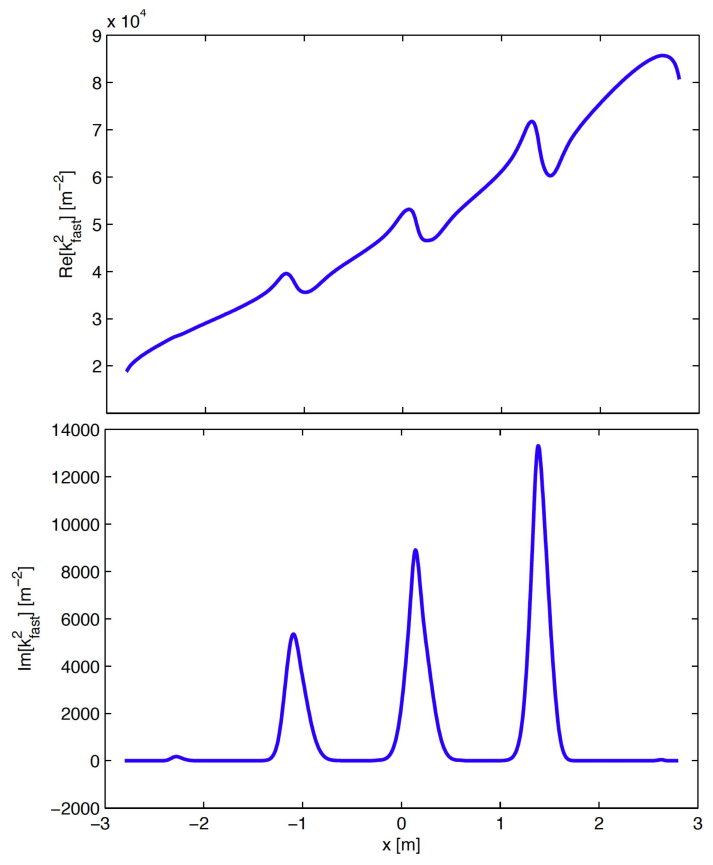

Figure 5: Fast wave dispersion root at $f=300 \mathrm{MHz}$ and $k_{/ /, o}=5 / m$ in a $D-T-(\alpha)-\left(D_{N B I}\right)$ DEMO plasma; $B_{o}=5.74 T$. The top curve shows the real part of the fast wave root; the bottom plot depicts the imaginary part in which ion cyclotron heating at the 6th, 7th and 8th harmonic is observed.

over velocity space so that the independent variables are spatial coordinates, the Fokker-Planck equation is necessarily solved in terms of constants of the motion. The distribution function of a given plasma species represented in terms of the constants of the motion (e.g. energy, magnetic moment) is, by definition, the same along the trajectory. However, because of the magnetic field inhomogeneity, the same distribution expressed in terms of its local velocity components $\left(v_{\perp}, v_{/ /}\right)$looks different depending on the location one looks at it (see Fig.6). Hence, interpretation of experimental data requires careful analysis: As diagnostics focus on different aspects of a same distribution, they may seemingly contradict but in truth corroborate one another.

The Coulomb collision operator for a uniform plasma is known. A convenient, symmetrical form is due to Landau (see e.g. $[1,2,18]$ ):

$$
\begin{gathered}
\sum_{s} C\left(F_{o a}, F_{o s}\right)=\nabla_{\vec{v}} \cdot \sum_{s} \vec{S}_{C}^{a / s} \\
\vec{S}_{C}^{a / s}=\frac{q_{a}^{2} q_{s}^{2} \ln \Gamma^{a / s}}{8 \pi \epsilon_{o}^{2} m_{a}} \int d \vec{v}^{\prime} \frac{u^{2} \overrightarrow{\overrightarrow{1}}-\vec{u} \vec{u}}{u^{3}}\left[\frac{F_{o a}}{m_{s}} \frac{\partial F_{o s}}{\partial \vec{v}^{\prime}}-\frac{F_{o s}}{m_{a}} \frac{\partial F_{o a}}{\partial \vec{v}}\right]
\end{gathered}
$$

in which 'a' refers to the species under examination and the sum is over all species 's' in the plasma; $\vec{u}$ is the relative velocity $\vec{v}-\vec{v}^{\prime}$. Since the species of type 'a' is one of the species in the sum, the collision operator is a non-linear integro-differential operator. If the species 'a' is a small minority, its selfcollisions can be neglected and the Fokker-Planck equation becomes a linear equation in $F_{o a}$, but if it is one of the main constituants the nonlinear collision operator has to be retained.

(a)
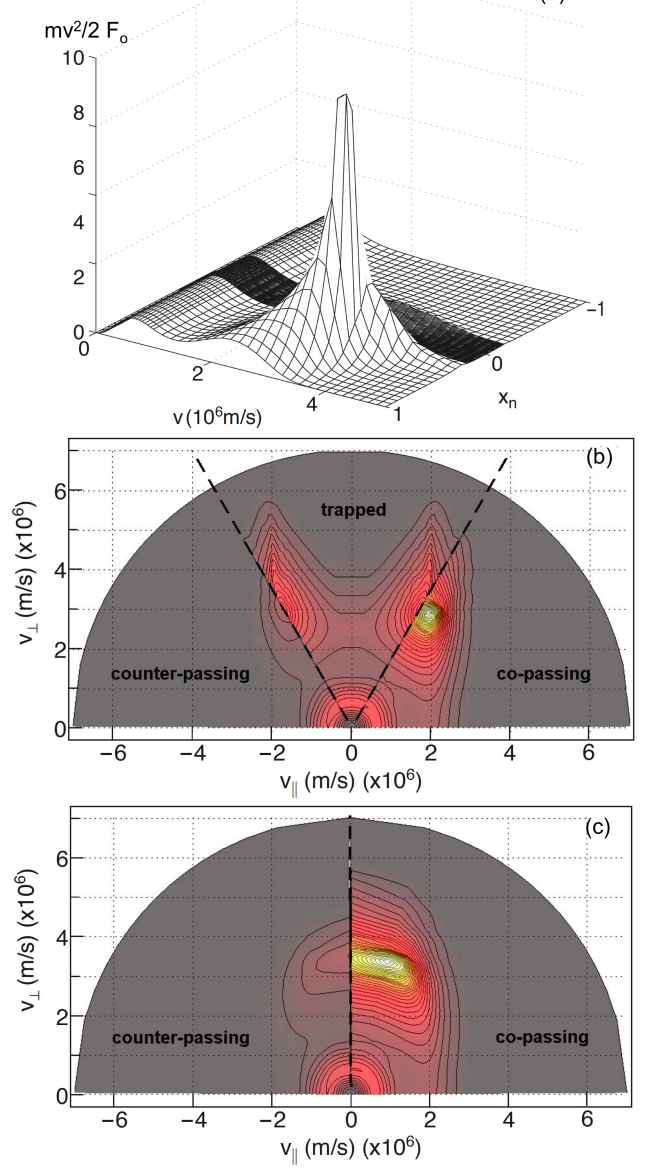

Figure 6: 3 representations of the same RF heated beam distribution energy density $\frac{m v^{2}}{2} F_{o}$ : (a) as a function of the constants of the motion velocity $v$ and (normalized) magnetic moment $x_{n}$, and as a function of $\left(v_{\perp}, v_{/ /}\right)$at (b) the low field side midplane and (c) the high field side midplane (see [23]).

Again, analytical expressions are available for the case the distribution function of the species ' $\mathrm{s}$ ' is Maxwellian, in which case the collision operator can be written in terms of the error function. In case $F_{o s}$ is isotropical, the integrals that need to be evaluated reduce to 1-dimensional integrals and in the fully anisotropical case the operator acting on $F_{\text {oa }}$ can be written in terms of the Rosenbluth potentials. The step from the uniform plasma collision operator $C$ to the operator $\langle C\rangle$ averaged over all fast aspects of the motion is a nontrivial step, the fully rigorous treat- 
ment of which is still awaited.

In view of the fact that the various species interact with each other collisionally, and that several types of species can simultaneously be heated by electromagnetic waves, a series of coupled Fokker-Planck equations rather than a single one should be solved. This can be done iteratively, taking the distributions obtained in the previous iteration to compute the collision operator in the current step. Provided convergence is reached, this allows accounting for the non-linear collision operator without making use of a non-linear system solver. Figure 7 shows a simplified 1-dimensional case in which it was assumed that all distributions are isotropic. It depicts an ITER example for the conditions foreseen for wave heating of the $D-T$ plasma during the activated phase of operation of the machine: the majority of $T$ ions is heated at its second harmonic cyclotron layer, while a minority of ${ }^{3} \mathrm{He}$ is simultaneously heated at its fundamental cyclotron resonance to help cranking up the fusion reactivity; unavoidably, the electrons are heated by Landau and TTMP damping.
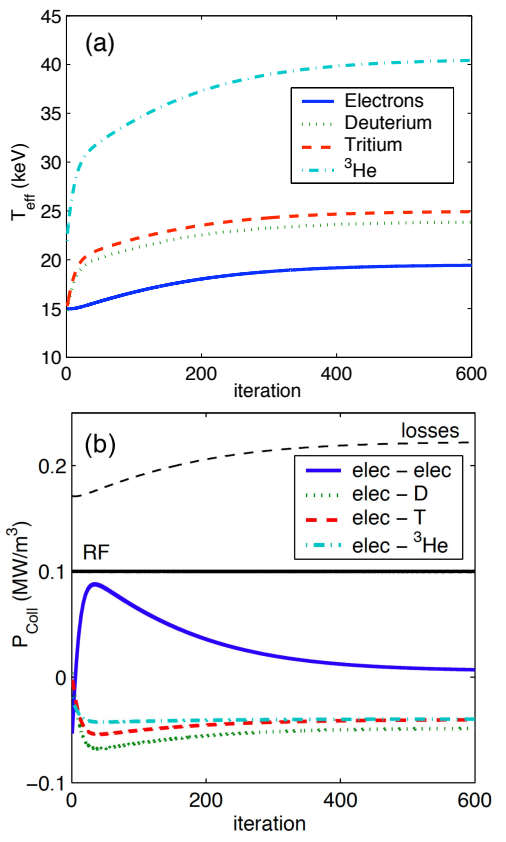

Figure 7: ITER $D-T-\left({ }^{3} \mathrm{He}\right)$ heating: (a) Effective temperatures and (b) electron power balance.

\section{II.G. A Note on Selfconsistency}

A rigorous treatment requires that the FokkerPlanck $(F P)$ and wave $(W)$ equations are solved simultaneously and on the same footing. Their intimate connection is exemplified by the 2 expressions of the absorbed power density:

$$
P_{a b s, F P}=\left.\frac{\partial}{\partial t}\left[\int d \vec{v} d \vec{x} \epsilon F_{o}\right]\right|_{R F}=\left.\int d \vec{v} d \vec{x} \epsilon \frac{\partial F_{o}}{\partial t}\right|_{R F}=
$$

$$
\begin{gathered}
\frac{1}{2} \operatorname{Re} \int d \vec{v} d \vec{x} \epsilon \nabla_{\vec{v}} \cdot \vec{a}_{R F}^{*} \int_{-\infty}^{t} d t^{\prime} \vec{a}_{R F} \cdot \nabla_{\vec{v}} F_{O} \\
=\frac{q}{2} \operatorname{Re} \int d \vec{v} d \vec{x} \vec{E}^{*} \cdot \vec{v} f_{R F}=\frac{q}{2} \operatorname{Re} \int d \vec{x} \vec{E}^{*} \cdot \vec{J}_{R F}=P_{a b s, W}
\end{gathered}
$$

in which $P_{a b s, F P}$ is the $\mathrm{RF}$ power density written in the way it is used in the Fokker-Planck equation (with $\epsilon$ the energy, and $\left.\frac{\partial F_{o}}{\partial t}\right|_{R F}$ the RF diffusion operator, and $P_{a b s, W}$ the RF power density as written in the wave equation, involving the RF perturbed current density $\vec{J}_{R F}$ and $f_{R F}$ the perturbed distribution function; $\vec{a}_{R F}=\frac{q}{m}\left[\vec{E}_{R F}+\vec{v} \times \vec{B}_{R F}\right]$ is the Lorentz force acceleration/decelaration caused by the small but rapidly varying electromagnetic field driven at frequency $\omega$. Formally writing down the above expression is immediate. To come up with practical expressions for the coefficients to be used in the wave and Fokker-Planck equations is less trivial, at least when the equations are truly treated on the same footing i.e. when the 2 problems posed in 6-dimensional phase space are solved removing 3 of the 6 independent variables to arrive at an equation in the remaining 3 variables using the same approximations for both equations. Getting the proper coefficients requires integrating (a) on the velocity space variables to obtain the dielectric response coefficients needed in the wave equation and (b) on the gyro, bounce and drift motions to find an expression for the quasi-linear diffusion operator. Ideally, the same elementary 'building blocks' are used and the relevant integrations are performed on them.

\section{SOME ASPECTS OF NONUNIFORM PLASMA MODELING}

\section{III.A. Mode Coupling [38-51]}

Before commenting on the particular issues brought about by the impact of the plasma inhomogeneities on the orbits of the particles and the challenges this leads to when trying to write down a rigorous expression for the dielectric response, a simplified problem is looked at first, namely that of the wave propagation in a tokamak in absence of a poloidal field i.e. where the guiding center orbits are assumed to simply being given by $\varphi(t)=\varphi\left(t_{o}\right)+v_{/ /}\left(t-t_{o}\right)$. Starting from Eq. (6), but retaining the full wave spectrum and toroidal curvature while assuming that the various species are Maxwellian and that the toroidal angle as well as the distance from midplane are ignorable variables (allowing to isolate individual $n$ toroidal modes and $k_{Z}$ ) yields

$$
\begin{gathered}
W=\omega \mu_{o}(2 \pi)^{3} \int d R R d v_{\perp} d v_{/ /} v_{\perp} \frac{F_{o}}{k T} \\
\sum_{N} \frac{\left[\int k_{R^{\prime}} L_{N}(\vec{F})\right]^{*}\left[\int k_{R} L_{N}(\vec{E})\right]}{N \Omega+k_{/ /} v_{/ /}-\omega}
\end{gathered}
$$


which is fully symmetrical w.r.t. the test function vector $\vec{F}$ and the electric field $\vec{E}$, guaranteeing a positive definite power density for a plasma in thermal equilibrium. To arrive at a practical expression one of the following 2 approaches is used:

- Assuming that $k_{\perp} \rho<<1$ so that the Bessel functions in the Kennel-Engelmann expressions can be approximated by a truncated Taylor series expansion around the origin, which upon realizing that

$$
\frac{d^{m}}{d R^{m}} \vec{E}(R)=\int d k_{R}\left(i k_{R}\right)^{m} \exp \left[i k_{R} R\right] \vec{E}_{k_{R}}
$$

allows to write down an expression for the dielectric response $W$ and the purely electromagnetic (curl) term to be used in the Galerkin form of the wave equation; it is customary to truncate the Taylor series at terms of second order in $k_{\perp} \rho$. Removing the differential operators from the test function vector components $\vec{F}$ by partial integrations allows to find the corresponding expression for the dielectric tensor, and the so obtained surface terms immediately provide the expression for the kinetic flux [38].

- In reality, $k_{\perp} \rho<<1$ is not satisfied for all modes that the plasma supports and thus that assumption should not be made if such modes are excited. Bernstein modes are finite temperature modes for which $k_{\perp} \rho \approx 1$ and even the cold plasma slow mode violates the smallness condition. Hence if short wavelength branches are excited - either directly at the plasma edge or at ion-ion hybrid layers [3] - a more rigorous treatment is needed to ensure the predicted fate of the shorter wavelength modes is correctly described. The easiest way to do this is to rewrite the Fourier integrals as discrete sums and to use locally constant base functions $\left[H\left(k_{R}-k_{R, i}\right)\right]\left[H\left(k_{R, i+1}-k_{R}\right)\right]$. The Galerkin form of the wave equation is hereby transformed into a system of linear equations allowing to find the electric field Fourier components in the discretized Fourier space.

Figure 8 shows an example of the integration of the 1D integrodifferential wave equation. The top figure depicts the perpendicular wave components. An incoming fast wave carries energy into the region of interest from the right. At the ion-ion hybrid layer at $R \approx 3 \mathrm{~m}$ mode conversion to the Bernstein wave takes place, although part of the fast wave energy simply tunnels through the confluence layers and makes it to the high field side (left on the plot) as a fast wave. Note that the Bernstein wave is efficiently absorbed, its amplitude having shrunk again to zero about $0.3 \mathrm{~m}$ towards the high field side. The bottom figure shows the corresponding
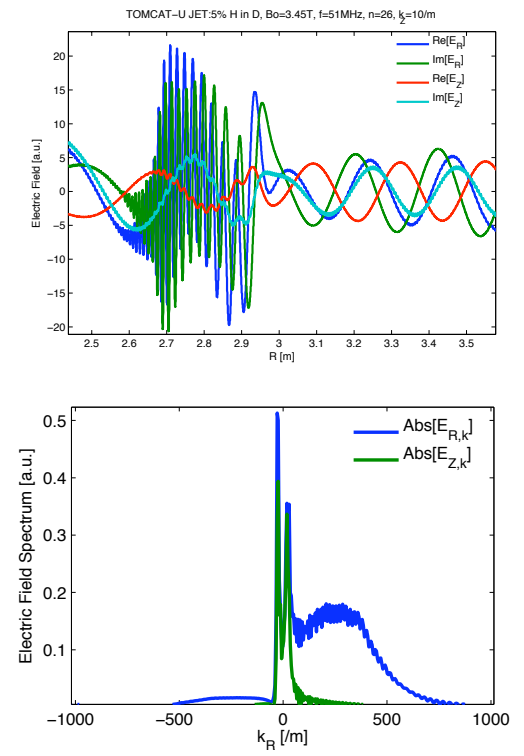

Figure 8: Bernstein wave excitation by the fast magnetosonic wave at the ion-ion hybrid layer: electric field components (top) and Fourier spectrum (bottom).

$k_{R}$ Fourier spectrum of the perpendicular electric field. The Bernstein wave is a backward, electrostatic wave: Its main field component is the component in the direction of the background gradient, and for a leftward propagating wave that carries energy from the confluence layer towards the high field side it is the $k_{R}>0$ spectrum that is significantly non-zero. The 2 peaks in the low $k_{R}$ part of the spectrum correspond to the incoming fast wave (highest amplitude for $k_{R}<0$ as the fast wave is a forward wave carrying energy in the same direction as the phase velocity) and the reflected wave (somewhat smaller peak, and in the $k_{R}>0$ region since the reflected wave necessarily carries less energy that the incoming wave).

In two dimensions poloidal as well as radial mode coupling occurs. Figure 9 gives an example of 2D wave equation modeling in which the geometry and the poloidal magnetic field has been accounted for. In this ITER example the short wavelength modes are not excited.

\section{III.B. Orbit topology [24-32]}

The motion of a charged particle in an axisymmetrical tokamak is characterized by 3 constants of the motion and by 3 periodic aspects of the motion. The 3 constants of the motion often used are the energy $\epsilon=\frac{m v^{2}}{2}$, the magnetic moment $\mu=\frac{m v_{\perp}^{2}}{2 B_{o}}$ and the toroidal angular momentum $P_{\varphi}=m R v_{\varphi}-q \Psi / 2 \pi$ ( $\varphi$ is the toroidal angle, $q$ the charge and $\Psi$ the poloidal magnetic flux) but suitable other sets of 3 independent functions of the customary 3 can equally well be used. In order of de- 


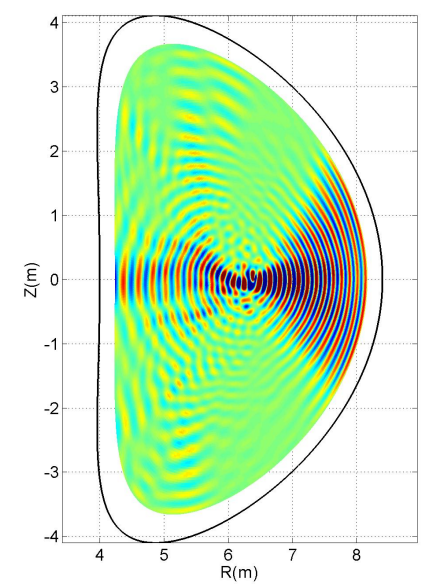

Figure 9: Poloidal electric field component for the RF heating scenario foreseen for the activated ITER phase; $3 \%{ }^{3} \mathrm{He}$ in a balanced $\mathrm{D}-\mathrm{T}$ plasma, $\mathrm{f}=53 \mathrm{MHz}$ and $B_{o}=5.3 T$.

creasing oscillation frequency, the 3 oscillatory aspects are the cyclotron motion, the bounce motion and the toroidal drift motion. Figure 10 gives a schematic view of the various oscillatory aspects of the motion for a trapped particle in a tokamak.

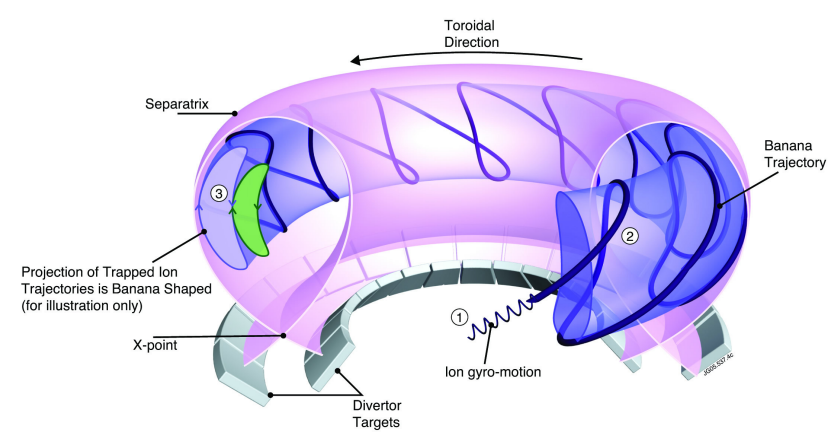

Figure 10: Schematic representation of the particle orbits in a tokamak (JET-EFDA figure JG05.537-4).

Even on a single particle level, adding the poloidal field to the description vastly changes the complexity of the wave-particle interaction problem since the guiding center orbits are now no longer on $R=c t$ surfaces but have become poloidally closed loops. Rather than sampling a unique value of the confining magnetic field, the guiding centers sample regions of varying toroidal field strength. Whereas in a uniform plasma a particle either is 'in resonance' or 'out of resonance' at all times, the resonances in inhomogeneous plasmas are localized i.e. the resonance condition is satisfied only locally at some positions along the orbit. The phase factor $\exp \left[i\left(N \Omega+k_{/ /} v_{/ /}-\omega\right)\left(t-t_{o}\right)\right]$ in the earlier men- tioned evolution equation for the particle energy is now generalized to an integral over $\vec{k}$ space of terms of the form $\exp [i \Theta(t)]$ in which $\Theta=-N \phi+\vec{k}(t) \cdot \vec{x}_{G C}(t)-\omega t$. In the neighbourhood of the resonance the phase in the exponential can be approximated by a truncated Taylor series expansion, $\Theta(t) \approx \Theta\left(t_{o}\right)+\dot{\Theta}\left(t_{o}\right)\left(t-t_{o}\right)+$ $\frac{1}{2} \ddot{\Theta}\left(t_{o}\right)\left(t-t_{o}\right)^{2}+\frac{1}{3 !} \dddot{\Theta}\left(t_{o}\right)\left(t-t_{o}\right)^{3}$. The corresponding exponential factor generally oscillates very quickly so that its integral does not accumulate a net contribution. Close to stationary phase points $(\dot{\Theta}=0)$ the phase variation slows down and the integral picks up a finite contribution. Figure 11 depicts the relevant integral for a regular stationary phase point $(\ddot{\Theta} \neq 0)$ and for a higher order stationary phase point $(\ddot{\Theta}=0)$. The former is representative for a standard resonance crossing while the latter is representative for a resonance at a turning point of the orbit, where 2 resonances merge (strictly, the higher order stationary phase point is a bit separated from the turning point: $v_{/ /}=0$ does not coincide with $v_{\theta}=0$ ). The linear line corresponding to the uniform plasma case for which the particle always stays in resonance is indicated as well.

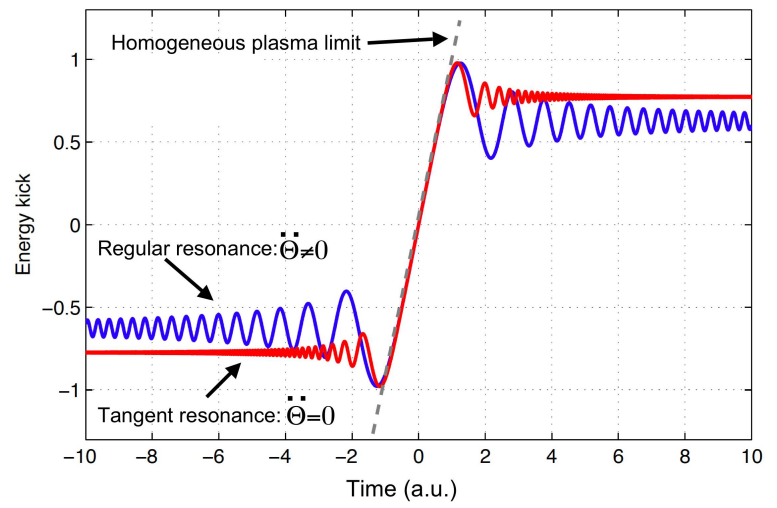

Figure 11: The energy kick felt by the particle along the orbit for resonance at a regular point $(\ddot{\Theta} \neq 0)$ and at a tangent resonance point $(\ddot{\Theta}=0)$.

In spite of the fact that energetic ions have guiding center orbits that deviate significantly from magnetic surfaces, the difference between the toroidal angular momentum $P_{\varphi}$ and the poloidal flux function $\Psi$ is often neglected ('zero drift' or 'zero banana width' approximation). Aside from the fact that this is an acceptable approximation in large enough machines or for low enough temperatures, the main motivation for this approximation is that it hugely simplifies the equations while keeping poloidal mode coupling and particle trapping/detrapping, two of the most important inhomogeneity effects, intact. Since the dielectric response written earlier was using the electric field at the guiding center rather than the particle position and since guiding centers stay on magnetic surfaces in the zero drift 
approximation, the parallel gradient can be written as an algebraic rather than as a differential operator when expressing the various quantities in terms of their (discrete) toroidal and poloidal Fourier series expansions:

$$
\begin{gathered}
\nabla_{/ /}=\frac{\cos \alpha}{|\partial \vec{x} / \partial \varphi|} \frac{\partial}{\partial \varphi}+\frac{\sin \alpha}{|\partial \vec{x} / \partial \theta|} \frac{\partial}{\partial \theta} \\
=\cos \alpha \frac{i n_{\text {tor }}}{R}+\sin \alpha \frac{i m_{p o l}}{|\partial \vec{x} / \partial \theta|}=i k_{/ /}
\end{gathered}
$$

for each individual poloidal mode $m_{\text {pol }}$ and toroidal mode $n_{\text {tor }} ; \alpha$ is the angle between the total magnetic field and the toroidal direction. The denominator resulting from the particle history integral is now no longer a constant and net resonant interaction only takes place at the poloidal angle that satisfies $N \Omega+k_{/ /} v_{/ /}=\omega$ in which the cyclotron frequency, the parallel wave number and the parallel velocity now all vary along the orbit. Although the density and temperature are constant along the zero-drift guiding center trajectory, poloidal mode coupling takes place because of the magnetic field and geometrical inhomogeneity the guiding center experiences along its orbit. This has one mild and one more important consequence:

- The mild consequency is that the perpendicular differential operator in the expression $L_{N}$ due to Kennel-Engelmann requires retaining the differential character in both independent perpendicular directions. The resulting expressions yield a double sum over poloidal modes, and differential operators in the direction perpendicular to the magnetic surfaces. For heating scenarios in which short wavelength branches are excited, a proper description of the poloidal coupling requires accounting for a very large number of poloidal modes and couplings, and a large number of radial grid points. In an axisymmetrical tokamak there is no toroidal coupling and thus a single sum on the toroidal mode spectrum remains; in a real tokamak - in which magnetic ripple occurs since a discrete number of toroidal magnetic field coils are installed - and in a stellarator, also the toroidal modes are coupled. Even in the zero drift limit, solving the wave equation in 2 or 3 dimensions requires powerful computers.

- Whereas the previous section involves supplementary bookkeeping but is not truly posing a problem, the fact that the parallel mode number appears in the resonant denominator gives rise to a fundamental problem: Whereas expression (6) is fully symmetrical in the test function vector $\vec{F}$ and the electric field $\vec{E}$ and guarantees positive definite and purely resonant absorption for Maxwellian distributions, which is what is physically expected, the now obtained expressions are symmetrical for what concerns the perpendicular operator but are asymmetrical for what concerns the parallel dynamics. As long as $k_{/ /}$is modest (as is typically the case for the fast wave), this is of little consequence. But for short wavelength branches, positive definite absorption for Maxwellian distributions is no longer guaranteed. A rigorous cure for that flaw requires a much more sophisticated model, as will be discussed in the next section.

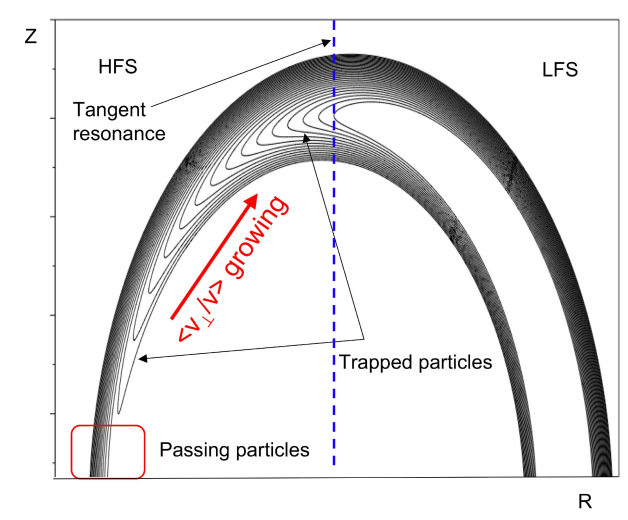

Figure 12: Schematic representation of the impact of cyclotron heating on a charged particle in a tokamak: The perpendicular energy of the particle gradually increases. Initially passing particles become trapped, their banana tip shifting towards the low field side when $v_{\perp}$ gradually grows. The interaction of the particle with the wave stops when the orbit no longer cuts the resonance. Just prior to that happening, 2 resonances merge, giving rise to efficient heating at the tangent resonance.

It was mentioned earlier that for not too energetic ions the Doppler shift term $k_{/ /} v_{/ /}$in the resonance condition $N \Omega+k_{/ /} v_{/ /}=\omega$ is a small correction to the cyclotron term. As the corresponding distribution is only significant in a restricted region of velocity space, it implies that the region where cyclotron interaction takes place is restricted in space as well: $\delta R / R \approx \delta\left(k_{/ /} v_{/ /}\right) / \omega$. Although the electrons are equally resonantly interacting with the field, the resonance condition is much less stringent on them since $k_{/ /} v_{/ /}=\omega$ is commonly satisfied in a wide region because of the modest steepness of the temperature profile. Consequently, it is fairly straightforward in the ion cyclotron frequency domain to ensure ion heating can only take places at a predetermined location but it is less evident to avoid the often unwanted electron heating. In big, hot and dense machines such as ITER $\mathrm{RF}$ waves have already lost a non-negligible fraction of their energy by electron Landau and TTMP damping before arriving at the cyclotron layer. 


\section{III.C. Bounce Motion, Tangent Resonance, ...}

Two approaches are commonly used to derive the wave equation (and in particular to find a suitable expression for the RF perturbed distribution function $f_{R F}$ ) and the Fokker-Planck equation (and in particular the quasilinear diffusion operator $\langle Q\rangle$ ). One is the very intuitive approach in which the governing Lorentz force can readily be recognized in the expressions and for which the link with straight magnetic field line uniform plasm theory is direct (see e.g. [26, 44, 43]). The other is more formal but more general and allows to benefit from the action-angle (Hamiltonian) formalism (see e.g. [33, 35, 36, 30]).

Practical expressions proposed by various authors tend to differ somewhat since different variables are chosen and different approximations are made. For wave equation studies (focussing on the fast dynamics), the trajectory integral is most intuitive and therefore most frequently adopted but for Fokker-Planck equation, the details of the fast dynamics are only indirectly relevant and all has to be expressed in terms of constants of the motion, hence tending to be closer to the action-angle technique which elegantly allows to retain the slow time scale physics while integrating away the fast phenomena by suitable averages over the various relevant oscillation periods. Kaufman showed, however, that the Hamiltonian description can equally be used to describe the fast scale physics. More importantly still, he stressed that a rigorous description of both aspects of the wave-particle interaction requires making the same approximations in both equations if one wants to describe the physics self-consistently. If applied rigorously, the path integral and action-angle methods are fully equivalent; for a somewhat more detailed discussion, see [34]. However, and in spite of Kaufman's visionary paper and presently available powerful computers, a fully rigorous description of the plasma heating process by electromagnetic waves is still awaited and a fully selfconsistent description based on a sufficiently rigorous footing is a project still to be tackled ...

The drift approximation and quasilinear approach make sense because of the vastly different time and spatial scales to describe the wave-particle interaction by first computing the zero order motion in absence of the rapid but small perturbation, and to account for the corrections relying on perturbation theory. In an axisymmetric tokamak in absence of perturbations, the particle motion can be described in terms of 3 constants of the motion $\vec{\Lambda}$ and 3 angles $\vec{\Phi}$ that describe the periodic aspects of the particle motion. Kaufman proposed to rely on action-angle variables but in the literature a wide variety of constants of the motion was successfully used. In contrast, the choice of the angles as used in the Hamiltonian theory is much more appealing than any other choice since these angles vary lin- early with time and thus time history integrals become trivial: Formally, the integrals are like those appearing in the uniform plasma case since - once functions only involving constants of the motion have been pulled out of time history integrals (since $\left.\frac{d}{d t}\right|_{o}=\frac{\partial}{\partial t}+\vec{\omega} \cdot \frac{\partial}{\partial \Phi}$ e.g. $f_{R F}=-\int d t^{\prime} \vec{a}_{R F} \cdot \nabla_{\vec{v}} F_{o}$ can simply be written as $\left.f_{R F}=-\frac{\partial F_{o}}{\partial \vec{\Lambda}} \cdot \int d t^{\prime} \dot{\Lambda}\right)$, the rapidly varying phase factor denoting all 4 oscillatory aspects of the driven response and particle motion is of the form $\vec{m} \cdot \vec{\Phi}(t)-\omega t$ in which $\vec{\Phi}(t)=\vec{\Phi}\left(t_{o}\right)+\vec{\omega}\left(t-t_{o}\right)$ and the gyro, bounce and toroidal drift frequencies $\vec{\omega}=\left(\omega_{g}, \omega_{b}, \omega_{d}\right)$ are only depending on the constants of the motion $\vec{\Lambda}$; the corresponding mode numbers are $\tilde{\vec{m}}=\left(m_{g}=-N, m_{b}, m_{d}=\right.$ $\left.n_{\text {tor }}\right)$ in which the bounce mode number $m_{b}$ should not be confused with the poloidal mode number $m_{p o l}$ but the other 2 mode numbers correspond to the cyclotron mode and the toroidal mode numbers. And so time history integrals simply yield factors of the form $\ldots /[\tilde{\vec{m}} \cdot \vec{\omega}-\omega]$ i.e. prescribe that waves and particles resonantly interact when the resonance condition $\tilde{\vec{m}} \cdot \vec{\omega}=\omega$ ( $\omega$ being the generator frequency) is satisfied.

A major simplification of the algebra comes from the identity $\vec{a}_{R F}=\frac{i}{\omega m}\left[\frac{d}{d t} \nabla_{\vec{v}}-\nabla_{\vec{x}}\right] q \vec{E} \cdot \vec{v}$ (see e.g. [5]) since it allows to write the various contributions of which the time history integrals needs to be found to come up with an appropriate expression for the dielectric response of the plasma to a rapidly oscillating electromagnetic wave in terms of $\dot{\epsilon}=q \vec{E} . \vec{v}$. For example

$$
f_{R F}=\sum_{j=1}^{3} \frac{i}{\omega m} \frac{\partial F_{o}}{\partial \Lambda_{j}}\left[-q \vec{E} \cdot \nabla_{\vec{v}} \Lambda_{j}+\int^{t} d t^{\prime} D_{\Lambda_{j}} \dot{\epsilon}\right]
$$

in which $D_{G} \ldots=\frac{d}{d t}\left[\nabla_{\vec{v}} G \cdot \nabla_{\vec{v}} \ldots\right]+\nabla_{\vec{v}} G \cdot \nabla_{\vec{x}} \ldots$, hereby essentially reducing the algebraic work to be done to describe the impact of an arbitrary distribution function $F_{o}(\vec{\Lambda})$ to the work needed for the case of an isotropic distribution. For a Maxwellian distribution, it can easily be shown that the net absorption of wave energy by a particle population is positive definite and that the interaction is resonant in nature:

$$
\left.\sum_{\tilde{\tilde{m}}, \tilde{\vec{m}} h}<\left.\left.q \vec{E} \cdot \vec{v}\right|_{\tilde{\vec{m}}^{\prime}} ^{*} \int_{\infty}^{t} d t^{\prime} q \vec{E} \cdot \vec{v}\right|_{\tilde{\vec{m}}}\right\rangle=\sum_{\tilde{\vec{m}}} \frac{\left.|q \vec{E} \cdot \vec{v}|_{\tilde{\vec{m}}}\right|^{2}}{i[\tilde{\vec{m}} \cdot \vec{\omega}-\omega]} .
$$

Making use of generating functions for the transformation between canonical variables and applying them to the action-angle $(\vec{J}, \vec{\Phi})$ variables proposed by Kaufman, one finds $D_{J_{i}}=m \frac{\partial}{\partial \Phi_{i}}$ where $m$ is the mass of the examined type of particles [34]. Whereas the toroidal angular momentum $P_{\varphi}$ and the magnetic moment $\mu$ are natural variables to use in the computations, the third Kaufman action - related to the surface enclosed by the poloidal closed drift orbit - is not very practical. Replacing it by the energy $\epsilon$ allows to find a compact operator to generalize the expression found for a 
Maxwellian distribution to that for an arbitrary distribution $F_{o}(\vec{\Lambda}): \frac{\partial F_{o}}{\partial \Lambda_{1}} \rightarrow \frac{\partial}{\partial \Lambda_{1}}+N \frac{\partial}{\partial \Lambda_{2}}+n_{\text {tor }} \frac{\partial}{\partial \Lambda_{3}}$ when $\vec{\Lambda}=\left(\frac{\epsilon}{\omega},-\frac{m v_{\perp}^{2}}{2 \Omega}, P_{\varphi}=m R v_{\varphi}-\frac{q \Psi}{2 \pi}\right)$ where $\Psi$ is the poloidal magnetic flux.

Of course, although the Hamiltonian method offers an elegant framework to do the required evaluations, its simplicity is somewhat misleading:

- The Fourier transformation of the work $q \vec{E} \cdot \vec{v}$ done by the electric field on the particles, written down only formally in Kaufman's paper is where the full complexity of the acceleration and deceleration of particles on their orbits through an inhomogeneous static magnetic field will show up. Happily, the vast difference in time response time of the various aspects of the motion allows to rely on asymptotic techniques to perform this step. First, the Fourier analysis is performed at a fixed time, and then the integrals along the orbits are evaluated. The Fourier transform of $q \vec{E} . \vec{v}$ is

$$
\left.q \vec{E} \cdot \vec{v}\right|_{\tilde{\vec{m}}}(\vec{\Lambda})=\frac{1}{(2 \pi)^{3}} \int d \vec{\Phi} q \vec{E} \cdot \vec{v} \exp [-i \tilde{\vec{m}} \cdot \vec{\Phi}] .
$$

Formally writing the electric field in terms of its $\vec{k}$ spectrum so that, analogously to the uniform plasma Eq. (2), the phase of $q \vec{E} \cdot \vec{v}$ is $\vec{k} \cdot \vec{x}_{G C}-N \phi-$ $\omega t$ ( $\mathrm{GC}=$ guiding center), it can readily be seen that the stationary phase points of the $\tilde{\vec{m}}$ Fourier component are given by the condition $\vec{k} \cdot \vec{v}_{G C}+$ $N \Omega=\tilde{\vec{m}} \cdot \vec{\omega}$ so that the global resonance condition $\tilde{\vec{m}} \cdot \vec{\omega}=\omega$ can be rewritten as $\vec{k} \cdot \vec{v}_{G C}+N \Omega=\omega$, which reduces to the familiar resonance condition $k_{/ /} v_{/ /}+N \Omega=\omega$ of the uniform plasma (and more in particular $\rho / L_{B_{o}}=0$ i.e. driftless) limit. It is not a trivial task to rigorously account for the drift orbit effects since perpendicular corrections not only have to be added to the generalized Kennel-Engelmann operator but they now enter the resonance condition as well.

- A supplementary difficulty comes from the fact that there are many thousands of bounce modes that need to be evaluated to describe wave-particle interaction accounting for the plasma inhomogeneity rigorously. This amounts to a significant increase of the computation time required to solve the equations.

\section{III.D. Decorrelation, Superadiabaticity [33-37,52-55]}

It was discussed earlier that the work $q \vec{E} . \vec{v}$ the electric field does on a charged particle is an oscillatory function of time i.e. can be written as a sum of terms proportional to a phase factor $\exp [i \Theta(t)]$. As the guiding center orbits in the drift approximation are closed poloidally, the particles cross every poloidal position many times every second (bounce frequency). Most of the contributions to the work are oscillatory in nature and cancel out when integrated over all fast time scales (gyro-, poloidal bounce and toroidal drift motion), yielding a zero net effect. Only the resonant contributions possibly give rise to a finite effect. That even these do not cancel on average, is not as evident as it may seem at first sight: In general, the number of cycles the work goes through in between 2 successive transits is not an integer number and thus the phase change is not a multiple of $2 \pi$ so the average work done over a longer period of time is the sum of 'energy kicks' with the same amplitude but at different phase. Assuming that the phase difference between 2 successive transits modulo $2 \pi$ is $\Delta \Theta$, it can readily be seen that for every particular phase at a given crossing, there is another crossing in a not too distant past that more or less cancels out the present contribution since $\Delta \Theta$ attains any value between 0 and $2 \pi$ with equal probability. And so, even if the particle gets an energy kick every time it crosses the resonance, the net effect of many crossings (typically a few thousand per second for standard ion temperatures in typical working conditions) is still zero ... Unless something breaks the pure periodicity and makes the particles somehow 'forget' about their encounters in the far past so that rather than a very large number of crossings being relevant, only the most recent ones are. Collionality does exactly that.

Let us consider the simplest possible 'Krook' collision operator $C(f)=\nu f$, where $\nu$ is the dominant collision frequency for the species considered, to discuss the principle: Whereas the fast dynamics of the waveparticle interaction is typically described by the Vlasov equation, a somewhat more careful examination of the evolution equation we started from shows that collisionality can strictly not be omitted when describing the fast time scale: the collision operator $C$ in that original equation acts on the full distribution $f=F_{o}+f_{R F}$ and not only on the slowly varying part $F_{o}$. Hence the Vlasov equation should be extended to contain a small but nonzero contribution, reflecting the rare but nonabsent collisions the particles undergo along their unperturbed orbits: $\left.\frac{d}{d t}\right|_{o} f_{R F}+\vec{a}_{R F} \cdot \nabla F_{o}=C\left(f_{R F}\right)$. Writing the time derivative along the trajectory in terms of the constants of the motion $\vec{\Lambda}$ and the angles $\vec{\Phi}$ and assuming that the perturbed distribution can be written as the product of a term only involving slow dynamics and a term involving fast dynamics i.e. $f_{R F}=$ $H(\vec{\Lambda}, t) \tilde{f}(\vec{\Lambda}, \vec{\Phi}, t)$, the fast and slow dynamics can be isolated: $\left[\left.H \frac{d}{d t}\right|_{o} \tilde{f}+\vec{a}_{R F} \cdot \nabla_{\vec{v}} F_{o}\right] / \tilde{f}=\left[-\frac{\partial}{\partial t} H+\nu H\right]$. Since the right hand side of this expression only contains slow dynamics (no fast period response), one can formally write that both sides of this equation independently have to be equal to a slowly varying function, 
$G=G(\vec{\Lambda}, t)$ which is negligibly small on the fast dynamics time scale, $G \approx 0$. It follows that the factor $H$ can to good approximation be evaluated explicitly: $H \propto \exp (\nu t)$. And so the Vlasov equation is supplemented with a 'switch-off' or 'phase memory loss' factor: $f=-\int_{-\infty}^{t} d t^{\prime} H^{-1} \vec{a}_{R F} . \nabla F_{o}$ which - in view of the result found for $H$ consistent with the Krook collision operator - is simply equivalent to the 'causality rule' which prescribes the frequency $\omega$ in the driven time response factor $\exp (i \omega t)$ and the resonant denominator $\left(N \Omega+k_{/ /} v_{/ /}-\omega\right.$ in the uniform plasma expression, or $\vec{\omega} \cdot \tilde{\vec{m}}-\omega$ in its drift approximation generalisation) to be replaced by $\omega+i \nu$. Whereas the collisional contribution is very small, it plays a crucial role in the evaluation of the time history integral when integrating over many crossings through a given point on the closed bounce orbit: It constitutes the 'memory loss' factor ensuring that a finite net contribution is obtained for the resonant contributions to the work done by the driven electric field on the particles.

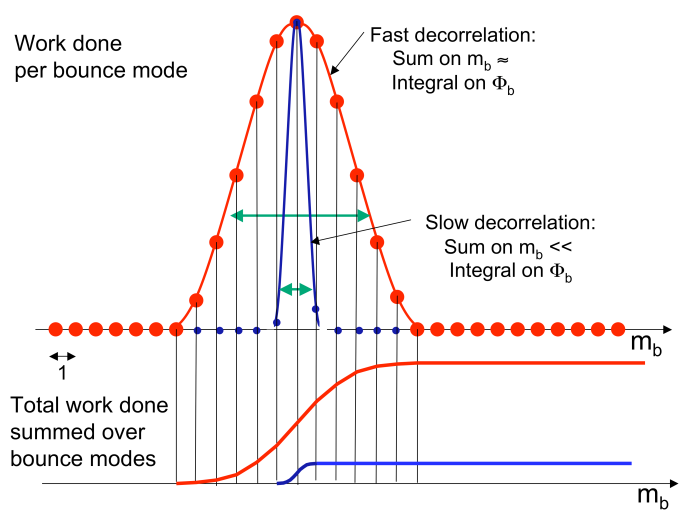

Figure 13: Schematic representation of the importance of decorrelation.

Why this is crucial and how it works can readily be seen in Fig. 13: due to the periodic nature of the bounce motion, the sum of the contributions over all bounce modes is only equivalent to the corresponding integral over (the stationary phase position) bounce angles if the collisional broadening of the resonance is wide enough. This brings out a subtle point in the analysis of the wave-particle interaction: Because of the large difference between the bounce and the gyrofrequency $\left(\omega_{b}<<\omega_{g}\right)$, it takes thousands of bounce modes to rigorously account for the magnetic field inhomogeneity i.e. the corresponding stationary phase points where the resonant interaction predominantly takes place $(\dot{\Theta}=0)$ are very closely spaced. Yet the discrete sum on the bounce modes cannot justifiably be replaced by a bounce integral unless the decorrelation time is short enough i.e. the collision frequency large enough. And so the very different time scale on which the gyro and bounce motions occur is crucial to restore the 'quasi-homogeneous' nature of localized resonances, while the decorrelation needs to be sufficiently fast to ensure that a net interaction takes place at these resonance locations when averaging over all the faster processes. In view of the typical collision frequencies in hot plasmas, collisions at first sight cannot cause a fast enough decorrelation to guarantee RF heating to have a net effect.

Fully accounting for the actual collision operator in the right hand side of the 'generalised' Vlasov equation is not at all a trivial task. Kasilov [53] did the exercise of examining more realistic collision operators and found that the 'switch-off' factor $H$ can to first approximation be taken to be $H=\exp \left[(t / \tau)^{n}\right]$ where $n=3$ for Cerenkov interaction (Landau damping and transit time magnetic pumping) and $n=5$ for cyclotron damping. He found that the decorrelation times $\tau$ are significantly shorter than the collison times $1 / \nu$, implying that particles 'lose' memory of their phase quickly enough for RF heating to be efficient in magnetic fusion devices. Although the details of the impact of the various decorrelation functions differs, the net effect is the same: the 'kick' particles receive when crossing the resonance is similar (see Fig. 14).

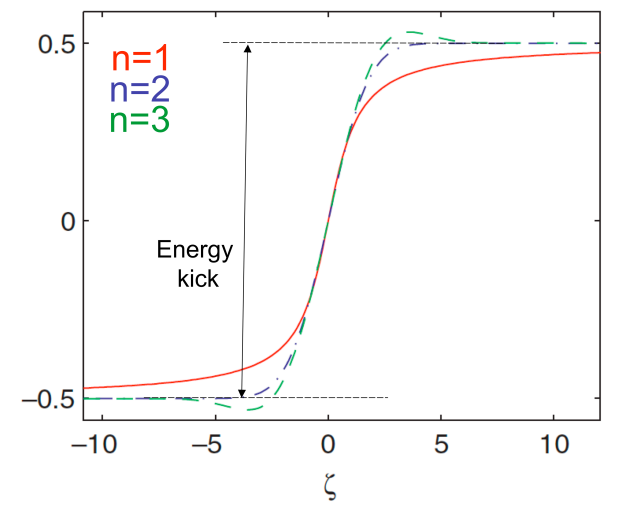

Figure 14: Integrated decorrelation functions for $n=$ $1,2,3 ; \zeta=\tau(\tilde{\vec{m}} \cdot \vec{\omega}-\omega)$ with $\tau$ the decorrelation time.

While for thermal particles it is thought that the details of the actual decorrelation are not too relevant (to the important exception of what happens near tangent resonance points where two closely spaced resonance points merge into a single one), for too energetic particles, however, the collisions may still be too unfrequent so that their net effective absorption is reduced. This regime is known as 'superadiabaticity'.

Not only collisions cause a randomization of the phase. Because of the non-integrability of the orbits and the non-linearity of the problem, stochastization 
takes place even if collisions would be absent when launching $\mathrm{RF}$ waves of a few MW in fusion relevant plasmas. The rich spectrum of modes, each contributing to the full wave-particle interaction and giving rise to fast phase variations of the total work done on the particles, is likely to trigger sufficient decorrelation.

\section{END NOTE}

With the dawn of powerful parallel computers, the degree of realism that can be reached when modeling the interaction of particles and waves in hot, magnetized, inhomogeneous plasmas contained in magnetic fusion experimental reactors is gradually increasing. Various techniques are available to highlight the study of specific aspects of the interaction. Even so, the problem to be tackled is challenging and a number of aspects are only starting to be touched upon.

One aspect of importance in the context of waveparticle interaction is the description of the opposite of wave heating, namely the onset of instabilities triggered by particle distributions: In present-day wave and Fokker-Planck descriptions, it is implicitly assumed that the RF fields are never powerful enough to make the factor $\frac{\partial F_{o}}{\partial \Lambda_{1}}+N \frac{\partial F_{o}}{\partial \Lambda_{2}}+n \frac{\partial F_{o}}{\partial \Lambda_{3}}$ that appears in the perturbed distribution $f_{R F}$ and hence in the expression for $\langle Q\rangle$ change sign, while experimentally it is well known that MHD modes can be triggered when RF heating is efficient.

Another - even more essential - aspect is the rigorous accounting of the bounce spectrum ensuring that the dielectric response is properly described, without artificially giving rise to damping that has to be rejected on physical grounds: While only the rigorous application of the procedure proposed by Kaufman guarantees the causality to be respected for all modes the plasma supports, no models based on this procedure are yet available.

\section{A COMMENT ON THE LIST OF REFERENCES}

Although most authors in the reference list comment on various subtopics treated in this text - making a clean separation impossible - the papers most relevant to read up on the general treatment have references [111], the wave equation is somewhat more the focus in [12-16] while the Fokker-Planck aspects are the main subject in [17-23]. Details on the orbit topology and its role in describing the wave-particle interaction can be found in [24-32]. The action-angle formalism is discussed in [33-37]. Comments on how to solve the relevant set of equations - with a focus on accounting for the realistic geometry - are given in [38-51] while the role of decorrelation is the key subject in [52-55].

\section{ACKNOWLEDGMENT}

The authors gladly thank Dr. P.U. Lamalle for the material he provided for this lecture.

\section{REFERENCES}

1. B. WEYSSOW, Kinetic Theory, these summer school proceedings

2. R. BALESCU, Transport Processes in Plasmas, North-Holland, Amsterdam (1988)

3. T.H. STIX, Waves in Plasmas, AIP, New York (1992)

4. B.D. FRIED and S.D CONTE, The Plasma Dispersion Function, Academic, New York (1961)

5. L.D. LANDAU \& E.M LIFSCHITZ, Course of Theoretical Physics, Pergamon, Oxford (1960)

6. R. KOCH, Phys. Lett. A 157, 399 (1991)

7. D. VAN EESTER, Plasma Phys. Contr. Fusion 35, 1309-1319 (1993)

8. D. VAN EESTER, Journal Plasma Phys. 54 1, pp. 31-58 (1995)

9. D. VAN EESTER, Journal Plasma Phys. 60 3, pp. 627-671 (1998)

10. D. VAN EESTER, Journal Plasma Phys. 65 5, pp. 407-452 (2001)

11. D. VAN EESTER, Plasma Phys. Contr. Fusion 47 5, pp. 459-481 (2005)

12. R. KOCH, Ion cyclotron, lower hybrid and Alfven wave heating, these summer school proceedings

13. E. WESTERHOF, Electron cyclotron waves, these summer school proceedings

14. P.U. LAMALlE, Phys. Lett. A 175, pp. 45-52 (1993)

15. P.U. LAMALLE, Non-local theoretical generalisation and tridimensional numerical study of the coupling of an ICRH antenna to a tokamak plasmas, LPP-ERM/KMS Laboratory Report 101, Brussels (1994)

16. P.U. LAMAlle, Plasma Phys. Contr. Fusion 39, 1409 (1997)

17. C.F. KENNEL and F. ENGELMANN, Phys. Fluids 9, 2377 (1966) 
18. C.F.F. KARNEY, Computer Physics Reports 4, North-Holland, Amsterdam, 1986 pp 183-244

19. J.D. GAFFEY, J. Plasma Phys. 16149 (1976)

20. R. KOCH, Fast particle heating, these summer school proceedings

21. E. WESTERHOF, Current Drive, these summer school proceedings

22. E. LERCHE et al., Plasma Phys. Contr. Fusion 50035003 (2008)

23. E. LERCHE et al., Plasma Phys. Contr. Fusion 51044006 (2009)

24. H. DE BLANK, Guiding center motion, these summer school proceedings

25. H. DE BLANK, Plasma equilibrium in tokamaks, these summer school proceedings

26. I.B. BERNSTEIN et al., Phys. Fluids 24, 108 (1981)

27. V.S. BELIKOV and Y. I. KOLESNICHENKO, Plasma Phys. 2461 (1982)

28. V.S. BELIKOV and Y. I. KOLESNICHENKO, Plasma Phys. Contr. Fusion 361703 (1994)

29. J. HEDIN, Ion Cyclotron Heating in Toroidal Plasmas, Ph.D. thesis, Royal Institute of Technology, Stockholm (2001)

30. J. HEDIN et al., Nucl. Fusion 42, pp. 527-540 (2002)

31. P.U. LAMALLE, Plasma Phys. Contr. Fusion 40 , 465-479 (1998)

32. P.U. LAMALLE, Plasma Phys. Contr. Fusion 48, 433-477 (2006)

33. A.N. KAUFMAN, Phys. Fluids 15 pp. 1063-1069 (1972)

34. D. VAN EESTER, Plasma Phys. Contr. Fusion 41, L23 (1999)

35. A. BECOULET et al., Phys. Fluids B 3, pp. 137$150(1991)$

36. A. BECOULET et al., Phys. Plasmas 1, pp. 29082925 (1994)

37. H.E. MYNICK, Nucl. Fusion 30357 (1990)

38. D. VAN EESTER et al., Plasma Phys. Contr. Fusion 40, 19491975 (1998)
39. L. VILLARD et al., Comput. Phys. Rep. 4, 95 (1986)

40. D. SMITHE et al., Phys. Rev. Lett. 60801 (1988)

41. O. SAUTER, Nonlocal Analyses of Electrostatic and Electromagnetic Waves in Hot, Magnetized, Nonuniform, Bounded Plasmas, LRP-457/92, Ecole Polytechnique Fédérale de Lausanne (1992)

42. M. BRAMBILLA, Nucl. Fusion 34, 1121 (1994)

43. M. BRAMBILlA, Plasma Phys. Contr. Fusion 41, 1 (1999)

44. E.F. JAEGER et al., Phys. Plasmas 81573 (2001)

45. E.F. JAEGER et al., Phys. Plasmas 13, 056101 (2006)

46. J.C. WRIGHT et al., Phys. Plasmas 11, 2473 (2004)

47. D.B. BATCHELOR, Integrated Simulation of Fusion Plasmas, Physics Today, 02/05, 35 (2005)

48. J. CARLSSON et al., Nucl. Fusion 37, 719 (1997)

49. J. CARLSON, ICRH Heating and Current Drive in Tokamaks, Ph.D. thesis, Royal Institute of Technology ISBN 97-7107-237-7 (1998)

50. T. HELLSTEN et al., Nucl. Fusion 44, pp. 892$908(2004)$

51. M. DIRICKX and B. WEYSSOW, J. Plasma Phys. 59211 (1998)

52. D. FAULCONER and R. LIBOFF, Phys. Fluids 151831 (1972)

53. S.V. KASILOV et al., Nucl. Fusion 302467 (1990)

54. D. VAN EESTER et al., Phys. Letters A, 218, pp. 70-79 (1996)

55. Y. LOUIS, Etude analytique et numérique de la réponse diélectrique non-locale d'un plasma de tokamak à une perturbation radiofréquence, tenant compte de la toroidicité et de la décorrelation, $\mathrm{PhD}$ thesis, Université Libre de Bruxelles, LPPERM/KMS Report 103 (1995) 